\title{
Estudo cefalométrico das alterações dos terços médio e inferior da face em pacientes com diferentes padrões respiratórios e faciais
}

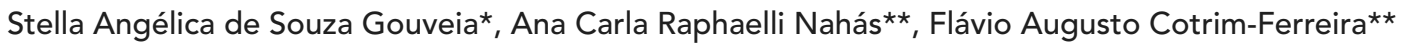

\section{Resumo}

Objetivos: avaliar a possível relação dos padrões respiratórios e dos tipos faciais com as grandezas da análise de Ricketts - CF-N.CF-A, Po-Or.ENA-ENP, CF-Go, CF-Xi.Po-Or, Xi-Pm, N-P.Go-M, A-NP, Po-Or.Na - e as grandezas da Nasofaringe e da Bucofaringe propostas por McNamara; dos tipos faciais com os padrões respiratórios e dos padrões respiratórios com as variáveis do Vert. Métodos: a amostra consistiu de 88 telerradiografias em norma lateral de brasileiros leucodermas, dos gêneros masculino e feminino, entre 7 e 13 anos (média de idade 10,5 anos), sendo 45 respiradores bucais e 43 nasais. O método cefalométrico computadorizado abrangeu a análise das grandezas para determinação do tipo facial, segundo Ricketts (Índice Vert). Aplicou-se a avaliação do erro do método pela fórmula de Dahlberg (média = 0,52), seguida das avaliações estatísticas teste " $t$ " de Student e Pearson product correlative coefficient test $(\mathrm{p}<0,05)$. Resultados e Conclusões: observou-se ausência de alterações estatisticamente significativas das variáveis quando relacionadas aos padrões respiratórios, e presença de diferenças estatisticamente significativas das variáveis Convexidade do ponto A, Altura posterior da face, Profundidade da maxila, Cone facial, Comprimento do corpo e Nasofaringe quando relacionadas aos tipos braqui e dolicofaciais. As variáveis Cone facial e Comprimento do corpo também apresentaram diferenças significativas entre os tipos braquifacial e mesofacial. A variável Cone facial apresentou diferenças significativas entre todos os tipos faciais; o tipo braquifacial apresentou correlação significativa $(\mathrm{p}=0,011)$ com o padrão de respiração nasal e a variável Profundidade facial apresentou diferença estatisticamente significativa $(p=0,028)$ quando relacionada aos padrões respiratórios.

Palavras-chave: Cefalometria. Crescimento craniofacial. Respiração bucal. Ricketts. McNamara.

* Mestre em Ortodontia pela Universidade Cidade de São Paulo - Unicid.

** Professores associados da disciplina de Ortodontia da Universidade Cidade de São Paulo - Unicid. 


\section{INTRODUÇÃO}

O relacionamento do padrão respiratório com o crescimento e o desenvolvimento das estruturas do complexo craniofacial tem sido objeto de interesse e controvérsias durante décadas ${ }^{8,11,12,26}$. Pesquisas ${ }^{6,7,28,30}$ demonstraram que apenas a herança genética não poderia definir o padrão de crescimento craniofacial. Desde então, fatores ambientais têm sido estudados, dentre eles o padrão respiratório, e sua relação com a morfologia dentofacial $16,18,21,24,31$.

Tonsilas faríngeas e palatinas são estruturas linfoides citadas na literatura ortodôntica como agentes obliteradores do espaço faringiano quando hipertrofiadas, dificultando a respiração nasal12,16,21,23,27,31. Quando a respiração bucal se estabelece, através de interações neuromusculares que mantêm a boca frequentemente aberta, pode provocar alterações morfológicas definidas na morfologia dentofacial. Caso essas alterações se expressem durante o crescimento, podem acarretar pressões musculares atípicas sobre o esqueleto craniofacial, repercutindo negativamente no desenvolvimento dessas estruturas ${ }^{15,18,22}$. Na infância e na adolescência, a respiração bucal prolongada, em razão da variedade de fatores etiológicos, pode estar relacionada aos pacientes portadores de face longa, atresia maxilar, palato ogival, mordida cruzada e retrognatismo mandibular ${ }^{4,18}$. Para se constatar se essas alterações morfológicas têm sido observadas nos respiradores bucais, métodos subjetivos e objetivos foram utilizados para determinar com clareza o padrão respiratório e, a partir daí, sua relação com o desenvolvimento dessas estruturas ${ }^{4,10,12,23,26,29}$.

Enquanto estudos concluíram que são limitados os dados que confirmam que a obstrução das vias aéreas é a responsável pela indução das alterações na morfologia craniofacial - ou que estas alterações são primárias, determinadas geneticamente $^{9}$-, outros compartilham do parecer de que se a respiração nasal é dificultada por algum motivo, instala-se um mecanismo compensatório de toda a estrutura facial, acarretando alterações morfológicas definidas nas regiões mediana e inferior da face ${ }^{6,7}$. Atualmente a obstrução respiratória tem sido citada como fator etiológico de importância no desenvolvimento da face e dos maxilares, onde atuaria apenas como agravante, porém não suficiente para alterar os fatores genéticos predeterminantes de cada indivíduo ${ }^{5}$.

Como ferramenta para esses estudos, a telerradiografia cefalométrica em norma lateral ${ }^{13,23}$ possibilita a avaliação dos vários graus de hipertrofia das tonsilas faríngeas, desde a sua ausência à sua presença obstrutiva, por meio da medição do espaço faringiano presente. Embora limitada pela imagem bidimensional das estruturas anatômicas complexas tridimensionais ${ }^{10}$, existe uma concordância de que as alterações cefalométricas mais comumente encontradas nos pacientes respiradores bucais são: retrusão mandibular, divergência dos planos oclusal e mandibular com rotação da mandíbula em sentido horário, aumento da altura facial anteroinferior e aumento do ângulo do plano mandibular ${ }^{26}$.

Baseado nessas investigações é que o presente estudo propõe-se a avaliar, por meio das análises cefalométricas computadorizadas em norma lateral, as possiveis alterações nos terços médio e inferior da face de brasileiros leucodermas, relacionando os padrões respiratórios e as variáveis cefalométricas das análises de Ricketts e McNamara; os tipos faciais e as variáveis cefalométricas relacionadas ao estudo; os tipos faciais e os padrões respiratórios; os padrões respiratórios e as variáveis do Índice Vert.

\section{MATERIAL E MÉTODOS}

A amostra consistiu de 88 telerradiografias em norma lateral - de brasileiros leucodermas na faixa etária dos 7 aos 13 anos (média de idade = 10,5 anos, d.p. = 18 meses), de ambos os gêneros, filhos ou netos de brasileiros descendentes de portugueses, espanhóis ou italianos - selecionadas nos arquivos do Departamento de Ortodontia da Universidade Cidade de São Paulo (Unicid). 
Foi estabelecido um protocolo prévio de seleção, por meio dos critérios de exclusão dos jovens com perdas precoces de dentes decíduos, histórico de hábitos bucais inadequados (sucção não-nutritiva), alterações patológicas (inserção de freios labial e lingual; macroglossia), tratamento ortodôntico prévio, histórico de cirurgias ou patologias associadas à obstrução nasofaríngea. Os 88 indivíduos selecionados, de um total de 120, foram submetidos à anamnese (por meio da observação clínica da postura dos lábios em repouso - selados ou afastados, úmidos ou ressecados) e avaliação fonoaudiológica (realizada pelo setor de Fonoaudiologia do Departamento de Ortodontia da Universidade Cidade de São Paulo - Unicid). Procedeu-se, então, ao preenchimento, em fichas apropriadas, dos critérios clínicos para a classificação do padrão respiratório: postura habitual dos lábios, postura corporal, tamanho e forma das narinas, controle reflexo dos músculos alares ${ }^{16}$, teste do espelho de Glatzel $^{1}$ e teste dos 3 minutos ${ }^{19}$.

Os três últimos testes foram decisivos na classificação do padrão respiratório dos indivíduos, sendo divididos em 45 respiradores bucais e 43 respiradores nasais.

As telerradiografias cefalométricas em norma lateral foram obtidas segundo a técnica de Broadbent $^{2}$, sendo utilizado um aparelho de raios $X$ (Panoura/10-CSU, Yoshida, Japão), regulado a $10 \mathrm{~mA}$ e $85 \mathrm{Kvp}$, com exposição de 0,8 segundo.

A elaboração dos cefalogramas foi realizada em um microcomputador Hewllet Packard CPV HP (Celeron 500Mhz, com HD de 20GB, 128MBRAM e um drive para discos) com o software Radiocef 2000 (Radio Memory, Belo Horizonte).

Os seguintes pontos cefalométricos (Fig. 1) foram utilizados: (1) Na (Násio), (2) Ba (Básio), (3) Po (Pório), (4) Or (Orbitário), (5) Pt (Pterigoide), (6) CF - Centro da face, (7) CC - Centro do crânio, (8) ENA - Espinha nasal anterior, (9) ENP - Espinha nasal posterior, (10) A - Subespinhal, (11) Pm - Protuberância mentoniana, (12) Pg - Pogônio, (13) Me - Mentoniano,
(14) Go - Gônio, (15) Xi - localizado no centro geométrico do ramo ascendente da mandíbula, (16) Dc - localizado no centro do côndilo sobre o plano Ba-Na, e (17) Gn - Gnátio.

A Bucofaringe (18) foi obtida linearmente a partir da interseção da borda posterior da língua com a borda inferior da mandíbula, até a parede posterior da faringe. A Nasofaringe (19) foi mensurada linearmente de um ponto médio da parede posterior do palato mole até a parede posterior da faringe. Todas as definições relacionadas aos pontos cefalométricos foram obtidas das análises de Ricketts $^{20}$ e McNamara ${ }^{13}$.

O traçado e a interpretação das grandezas cefalométricas foram utilizados na avaliação das estruturas craniofaciais numa perspectiva lateral, nos planos vertical e anteroposterior. As variáveis lineares nomeadas de Convexidade do ponto A, Altura posterior da face, Comprimento do corpo, Nasofaringe e Bucofaringe (Fig. 2) e as angulares Altura maxilar, Plano palatal, Posição do ramo, Profundidade maxilar e Cone facial (Fig. 3) foram selecionadas dentro do espectro de medidas preconizadas pelas análises de Ricketts e McNamara. Outras cinco variáveis - Eixo facial, Profundidade da face, Ângulo do plano mandibular, Altura inferior da face e Arco mandibular - representam o Índice Vert ${ }^{20}$ para determinação do padrão facial dos indivíduos da amostra (Fig. 4).

Após as mensurações das grandezas e o cálculo do índice Vert para a determinação do tipo facial de cada paciente, foi aplicada a análise da precisão do método cefalométrico pelo erro sistemático e pela expressão de Dahlberg ${ }^{3}$.

Das análises de significância estatística para a verificação da existência de diferenças - (a) entre os padrões respiratórios e 10 variáveis cefalométricas; (b) entre os tipos faciais e 10 variáveis cefalométricas; e (c) entre os padrões respiratórios e as variáveis do Vert -, foi aplicado o teste $t$ de Student ${ }^{25}$. Para as correlações dos padrões respiratórios e os tipos faciais, foi aplicado o Pearson product correlative coefficient test $t^{17}$. 


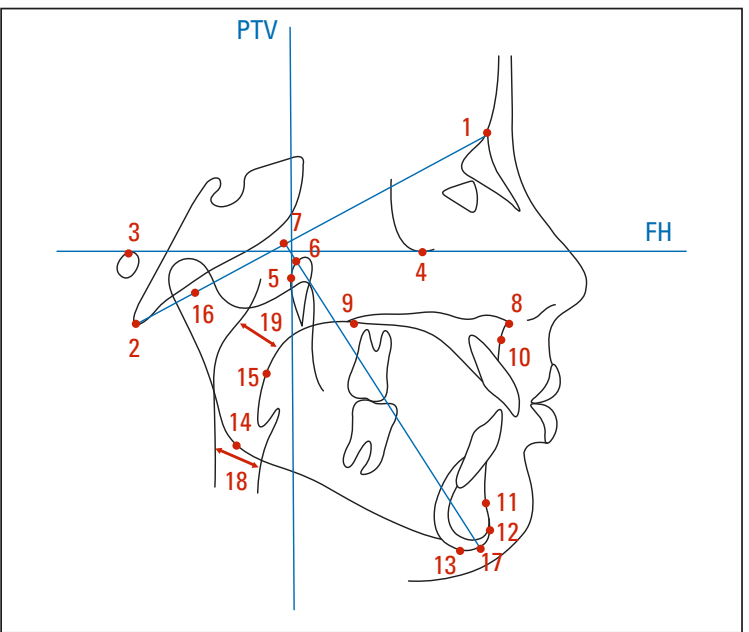

FIGURA 1 - Pontos cefalométricos: (1) Na - Násio, (2) Ba - Básio, (3) Po - Pório, (4) Or - Orbitário, (5) Pt - Pterigoide, (6) CF - Centro da face, (7) CC - Centro do crânio, (8) ENA - Espinha nasal anterior, (9) ENP - Espinha nasal posterior, (10) A - Subespinhal, (11) Pm - Protuberância mentoniana, (12) Pg - Pogônio, (13) Me - Mentoniano, (14) Go - Gônio, (15) $\mathrm{Xi}$ - localizado no centro geométrico do ramo ascendente da mandíbula), (16) Dc - localizado no centro do côndilo sobre o plano Ba-Na, (17) Gn-Gnátio, (18) Bucofaringe e (19) Nasofaringe.

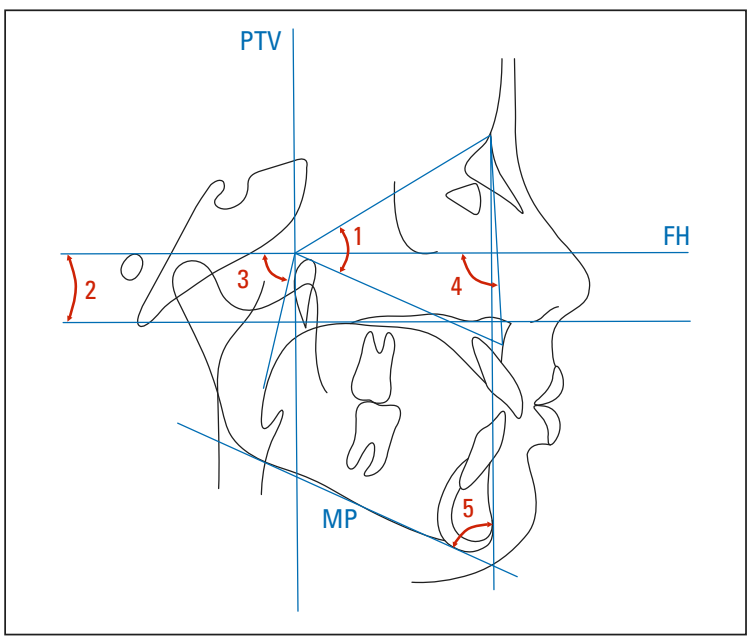

FIGURA 3 - Medidas angulares: (1) Altura maxilar - CF-N.CF-A; (2) Plano palatal - Po-Or.ENA-ENP; (3) Posição do ramo - CF-Xi.Po-Or; (4) Profundidade maxilar - Po-Or.NA e (5) Cone facial - N-P.Go-Me.

\section{RESULTADOS}

A tabela 1 apresenta as médias encontradas nas análises do erro sistemático e do erro do método pela expressão de Dahlberg (média $=0,52$ ), denotando confiabilidade nos resultados obtidos com o método cefalométrico.

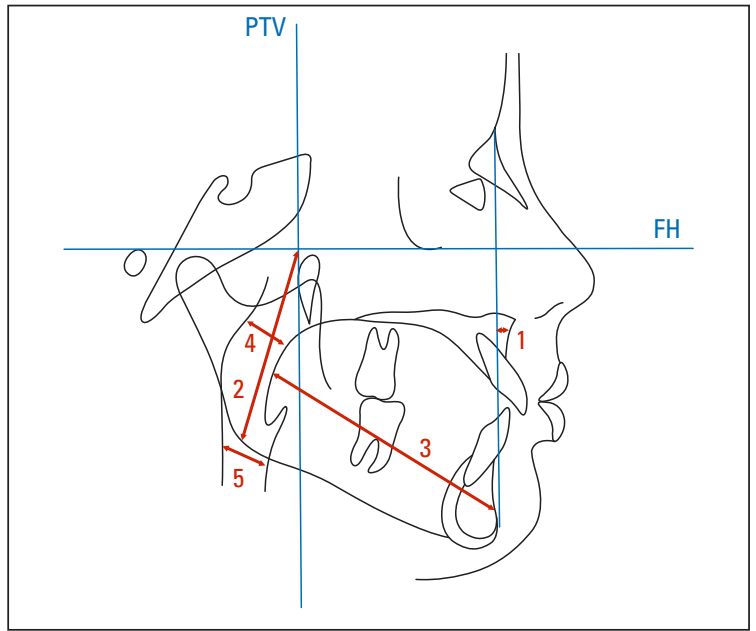

FIGURA 2 - Medidas lineares: (1) Convexidade do ponto A - A-NP; (2) Altura posterior da face - CF-Go; (3) Comprimento do corpo-Xi-Pm; (4) Nasofaringe e (5) Bucofaringe.

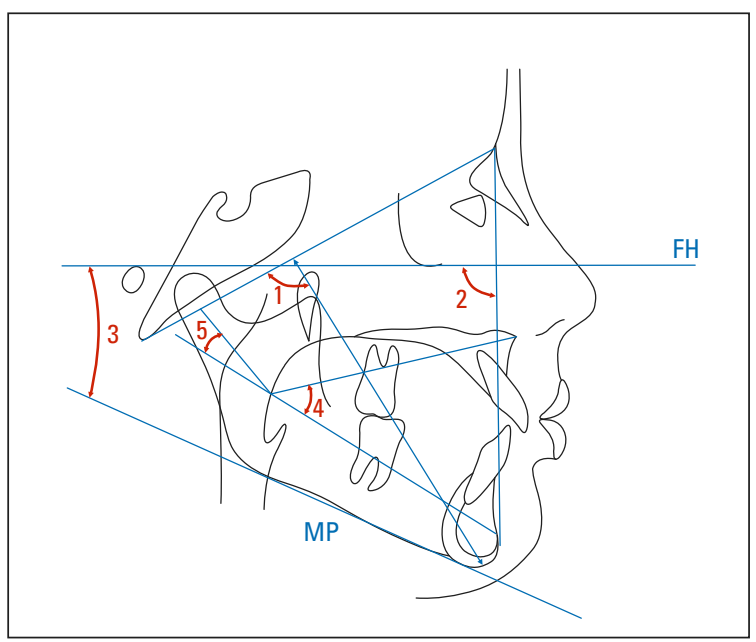

FIGURA 4 - Índice Vert: (1) Eixo facial - Pt-Gn. Ba-Na; (2) Profundidade da face-N-P.Po-Or; (3) Ângulo do plano mandibular - Go-Me.Po-Or; (4) Altura inferior da face - Xi-ENA.Xi-Pm e (5) Arco mandibular-Xi-Dc.Xi-Pm.

$\mathrm{Na}$ tabela 2, constatou-se que em nenhum caso ocorreu significância estatística ( $p>0,05)$, demonstrando que o padrão respiratório não está relacionado a nenhuma das grandezas estudadas.

Na tabela 3, os tipos braquifacial e dolicofacial relacionaram-se significativamente $(\mathrm{p}<0,05)$ 
TABELA 1 - Erro Sistemático e Dahlberg.

\begin{tabular}{|c|c|c|c|c|c|c|c|c|}
\hline & $\begin{array}{l}\text { C.PONTO A } \\
(\mathrm{mm})\end{array}$ & $\begin{array}{l}\text { P.FACIAL } \\
\text { (graus) }\end{array}$ & $\begin{array}{c}\text { A.E.FACIAL } \\
\text { (graus) }\end{array}$ & $\begin{array}{l}\text { C.FACIAL } \\
\text { (graus) }\end{array}$ & $\begin{array}{c}\text { P.MAXILA } \\
\text { (graus) }\end{array}$ & $\begin{array}{c}\text { A.MAXILAR } \\
\text { (graus) }\end{array}$ & $\begin{array}{c}\text { PPALATAL } \\
\text { (graus) }\end{array}$ & $\begin{array}{c}\text { A.P.MAND. } \\
\text { (graus) }\end{array}$ \\
\hline Erro Sistemático & 0,39 & 0,33 & 0,52 & 0,43 & 0,58 & 0,67 & 0,54 & 0,42 \\
\hline d.p. & 0,37 & 0,29 & 0,40 & 0,39 & 0,49 & 0,60 & 0,45 & 0,35 \\
\hline \multirow[t]{2}{*}{ Dahlberg } & 0,38 & 0,31 & 0,46 & 0,41 & 0,53 & 0,63 & 0,49 & 0,39 \\
\hline & $\begin{array}{l}\text { A.F.POST. } \\
\text { (mm) }\end{array}$ & $\begin{array}{l}\text { P. RAMO } \\
\text { (graus) }\end{array}$ & $\begin{array}{l}\text { A.MAND } \\
\text { (graus) }\end{array}$ & $\begin{array}{l}\text { C.CORPO } \\
(\mathrm{mm})\end{array}$ & $\begin{array}{c}\text { AFAl } \\
\text { (graus) }\end{array}$ & $\begin{array}{c}\text { NF } \\
(\mathrm{mm})\end{array}$ & $\begin{array}{c}\text { OF } \\
(\mathrm{mm})\end{array}$ & \\
\hline Erro Sistemático & 0,50 & 0,68 & 1,40 & 0,49 & 0,50 & 0,27 & 0,17 & \\
\hline d.p. & 0,42 & 0,63 & 2,17 & 0,51 & 0,39 & 0,45 & 0,38 & \\
\hline Dahlberg & 0,46 & 0,65 & 1,80 & 0,49 & 0,45 & 0,37 & 0,29 & \\
\hline
\end{tabular}

TABELA 2 - Distribuição da amostra por padrões respiratórios (Bucal e Nasal), relacionados às grandezas cefalométricas Convexidade do ponto $A$, Altura maxilar, Plano palatal, Altura facial posterior, Posição do ramo, Profundidade da maxila, Cone facial, Comprimento do corpo, Nasofaringe e Bucofaringe - Teste t de Student.

\begin{tabular}{|c|c|c|c|c|c|c|c|}
\hline \multirow{2}{*}{\multicolumn{2}{|c|}{ VARIÁVEIS }} & \multicolumn{2}{|c|}{ BUCAL } & \multicolumn{2}{|c|}{ NASAL } & \multirow[b]{2}{*}{$\mathbf{p}$} & \multirow{2}{*}{ sig. } \\
\hline & & média & d.p. & média & d.p. & & \\
\hline C. Ponto A & $(\mathrm{mm})$ & 4,33 & 2,64 & 4,22 & 2,67 & 0,837 & n.s. \\
\hline A. Maxilar & (graus) & 56,93 & 3,17 & 56,88 & 3,50 & 0,532 & n.s. \\
\hline P. Palatal & (graus) & 2,00 & 3,83 & 2,30 & 3,26 & 0,698 & n.s. \\
\hline A. F. Post. & $(\mathrm{mm})$ & 60,86 & 4,76 & 59,32 & 4,31 & 0,116 & n.s. \\
\hline P. Ramo & (graus) & 75,10 & 3,81 & 73,95 & 11,11 & 0,516 & n.s. \\
\hline P. Maxila & (graus) & 91,00 & 3,73 & 92,32 & 3,31 & 0,081 & n.s. \\
\hline C. Facial & (graus) & 64,81 & 3,81 & 65,02 & 3,46 & 0,790 & n.s. \\
\hline C. Corpo & $(\mathrm{mm})$ & 70,88 & 4,59 & 70,22 & 3,94 & 0,476 & n.s. \\
\hline Nasofaringe & $(\mathrm{mm})$ & 8,76 & 3,24 & 8,40 & 2,95 & 0,588 & n.s. \\
\hline Bucofaringe & $(\mathrm{mm})$ & 12,42 & 3,41 & 12,35 & 4,15 & 0,928 & n.s. \\
\hline
\end{tabular}

com seis das variáveis estudadas: Convexidade do ponto A ( $\mathrm{p}=0,004)$, Altura da face posterior ( $\mathrm{p}$ $=0,006)$, Profundidade da maxila ( $\mathrm{p}=0,007)$, Comprimento do corpo $(\mathrm{p}=0,026)$, Cone facial ( $p=0,000)$ e Nasofaringe $(p=0,015)$. Os tipos braquifacial e mesofacial relacionaram-se significativamente com as variáveis Cone facial $(\mathrm{p}=$ $0,003)$ e Comprimento do corpo $(p=0,042)$. A variável Cone facial também se relacionou significativamente entre os tipos mesofacial e dolicofacial $(\mathrm{p}=0,000)$.

A tabela 4 apresenta a distribuição da amostra pelos padrões respiratórios e tipos faciais, necessários para a realização da análise de correlação, que revelou correlação significativa $(p=0,011)$ entre o padrão respiratório nasal e o tipo braquifacial.

$\mathrm{Na}$ tabela 5 , ocorreram diferenças significativas ( $p=0,028)$ da variável profundidade facial quando relacionada aos padrões respiratórios.

\section{DISCUSSÃO}

Sabe-se que o padrão respiratório é de grande importância no diagnóstico e planejamento ortodônticos, assim como na estabilidade dos 
TABELA 3 - Distribuição da amostra pelos tipos faciais (Braquifacial-B, Mesofacial-M e Dolicofacial-D), relacionados às grandezas cefalométricas Convexidade do ponto A, Altura maxilar, Plano palatal, Altura facial posterior, Posição do ramo, Profundidade da maxila, Cone facial, Comprimento do corpo, Nasofaringe e Bucofaringe - Teste t de Student.

\begin{tabular}{|c|c|c|c|c|c|c|c|c|c|c|c|c|c|}
\hline \multirow{2}{*}{\multicolumn{2}{|c|}{ VARIÁVEIS }} & \multicolumn{2}{|c|}{ B } & \multicolumn{2}{|c|}{$\mathbf{M}$} & \multicolumn{2}{|c|}{ D } & \multicolumn{2}{|c|}{$\mathrm{BM}$} & \multicolumn{2}{|c|}{ BD } & \multicolumn{2}{|c|}{ MD } \\
\hline & & média & d.p. & média & d.p. & média & d.p. & $\mathrm{p}$ & sig. & $p$ & sig. & $p$ & sig. \\
\hline C. Ponto A & $(\mathrm{mm})$ & 2,92 & 1,71 & 4,10 & 2,63 & 5,14 & 2,77 & 0,098 & n.s. & 0,004 & * & 0,109 & n.s. \\
\hline A. Maxilar & (graus) & 55,60 & 3,88 & 56,56 & 3,14 & 57,42 & 3,14 & 0,338 & n.s. & 0,079 & n.s. & 0,257 & n.s. \\
\hline P. Palatal & (graus) & 2,97 & 3,85 & 2,26 & 3,14 & 1,62 & 3,81 & 0,475 & n.s. & 0,241 & n.s. & 0,443 & n.s. \\
\hline A. F. Post. & $(\mathrm{mm})$ & 62,66 & 4,99 & 60,36 & 3,74 & 58,55 & 4,71 & 0,066 & n.s. & 0,006 & * & 0,076 & n.s. \\
\hline P. Ramo & (graus) & 76,14 & 4,15 & 75,63 & 2,85 & 74,60 & 4,51 & 0,601 & n.s. & 0,244 & n.s. & 0,251 & n.s. \\
\hline P. Maxila & (graus) & 93,34 & 3,04 & 91,91 & 3,56 & 90,51 & 3,53 & 0,159 & n.s. & 0,007 & * & 0,101 & n.s. \\
\hline C. Facial & (graus) & 68,18 & 3,40 & 65,50 & 2,72 & 62,64 & 3,11 & 0,003 & * & 0,000 & * & 0,000 & * \\
\hline C. Corpo & $(\mathrm{mm})$ & 72,68 & 3,58 & 70,43 & 3,72 & 69,63 & 4,85 & 0,042 & * & 0,026 & * & 0,436 & n.s. \\
\hline Nasofaringe & $(\mathrm{mm})$ & 10,18 & 2,90 & 8,54 & 2,80 & 7,82 & 3,26 & 0,054 & n.s. & 0,015 & * & 0,323 & n.s. \\
\hline Bucofaringe & (mm) & 12,35 & 4,03 & 12,08 & 3,85 & 12,74 & 3,62 & 0,813 & n.s. & 0,734 & n.s. & 0,464 & n.s. \\
\hline
\end{tabular}

TABELA 4 - Distribuição dos indivíduos da amostra pelos padrões respiratórios (Nasal e Bucal) e tipos faciais (Braquifacial-B, Mesofacial-M e Dolicofacial-D).

\begin{tabular}{ccccc} 
AMOSTRA & \multicolumn{2}{c}{ NASAL } & \multicolumn{2}{c}{ BUCAL } \\
& $n^{0}$ & $\%$ & $n^{0}$ & $\%$ \\
\hline B & 13 & 30,23 & 4 & 8,89 \\
M & 17 & 39,54 & 20 & 44,44 \\
D & 13 & 30,23 & 21 & 46,67 \\
\hline
\end{tabular}

resultados obtidos. Quando a respiração sofre alterações significativas, essas podem repercutir de maneira negativa nos diferentes estágios do desenvolvimento facial, assim como no desenvolvimento da oclusão $0^{4,7,11,12,14,15,18,27,30,31}$. A avaliação e observação das estruturas do complexo craniofacial nesses diferentes estágios, por meio da telerradiografia em norma lateral, tem demonstrado ser um instrumento eficiente no diagnóstico e planejamento ortodônticos $4,8,9,10,12,13,20,23,24,26,30$.

TABELA 5 - Distribuição da amostra pelos padrões respiratórios (Bucal e Nasal) relacionados às variáveis cefalométricas do índice Vert: Altura facial inferior, Profundidade facial, Eixo facial, Ângulo do plano mandibular e Arco mandibular - teste t de Student.

\begin{tabular}{|c|c|c|c|c|c|c|c|}
\hline \multirow{2}{*}{ VARIÁVEIS } & & \multicolumn{2}{|c|}{ BUCAL } & \multicolumn{2}{|c|}{ NASAL } & \multirow{2}{*}{$\mathbf{P}$} & \multirow{2}{*}{ sig. } \\
\hline & & média & d.p. & média & d.p. & & \\
\hline A. F. Inf. & (graus) & 46,30 & 4,75 & 44,62 & 4,40 & 0,089 & n.s. \\
\hline P. Facial & (graus) & 86,64 & 2,82 & 88,01 & 2,93 & 0,028 & $*$ \\
\hline Eixo Facial & (graus) & 85,36 & 4,28 & 86,48 & 4,12 & 0,214 & n.s. \\
\hline A. P. Mand. & (graus) & 28,43 & 4,53 & 26,93 & 4,99 & 0,144 & n.s. \\
\hline Arco Mand. & (graus) & 25,42 & 5,40 & 27,50 & 6,12 & 0,095 & n.s. \\
\hline
\end{tabular}


Baseado nas informações obtidas a partir desses exames,verificou-se que a tabela 2 revela a ausência de diferenças estatisticamente significativas entre as variáveis cefalométricas e os padrões respiratórios, resultados esses concordantes com outros estudos ${ }^{5,9,29}$ que também não verificaram diferenças significativas nas relações entre os padrões respiratórios e as grandezas envolvidas no estudo da morfologia facial. Contrariando esses resultados, várias pesquisas ${ }^{11,13,14,18,21,24,27,31}$ constataram que as grandezas cefalométricas relacionadas sofreram influência dos padrões respiratórios.

$\mathrm{Na}$ tabela 3, observa-se que seis dessas variáveis apresentam diferenças significativas com os tipos faciais: Convexidade do ponto A, Altura facial posterior, Profundidade maxilar, Cone facial, Comprimento do corpo e Nasofaringe. Essas variáveis apresentam diferenças significativas entre os tipos dolicofacial e braquifacial, sendo que a variável Cone facial também apresenta diferenças significativas entre os tipos mesofacial e dolicofacial, e entre os tipos mesofacial e braquifacial. A variável Comprimento do corpo, além de se relacionar significativamente entre dolicofaciais e braquifaciais, também apresenta resultados significativos entre os grupos braquifacial e mesofacial. Resultados similares não constataram diferenças significativas dessas variáveis entre os diferentes tipos faciais ${ }^{20,27}$. Entretanto, resultados contrários $^{9,10,18}$ não apontaram diferenças estatisticamente significativas entre essas variáveis e os diferentes tipos faciais, visto que, nos estudos revisados, as variáveis mais empregadas nessas relações são a AFAI e o Ângulo do plano mandibular $5,8,11,26$.

Ao analisar a tabela 4 , verifica-se a distribuição dos indivíduos respiradores bucais e nasais nos diferentes tipos faciais. O grupo dos 43 respiradores nasais é constituído por 13 indivíduos braquifaciais $(30,23 \%), 17$ mesofaciais $(39,54 \%)$ e 13 dolicofaciais (30,23\%). Já no que se refere ao grupo dos 45 respiradores bucais, relacionaram-se 4 indivíduos braquifaciais (8,89\%), 20 mesofaciais $(44,44 \%)$ e 21 dolicofaciais $(46,67 \%)$. Na verifi- cação da existência de correlação entre os tipos faciais e os padrões respiratórios, conclui-se que os padrões respiratórios apresentam diferenças fortemente significativas com o tipo braquifacial, sendo que, nesse tipo facial, foi evidenciado o predomínio dos respiradores nasais. Encontrou-se anuência na literatura ${ }^{6,14,18,22,24,30,31}$ com correspondências entre os padrões respiratórios e os tipos faciais. Foi verificada que a obstrução respiratória pode ser encontrada nos diferentes tipos faciais, e que o diagnóstico da obstrução nasal é fundamental nos pacientes com más oclusões, principalmente os dolicofaciais ${ }^{4}$. Contrariamente aos nossos resultados, outras avaliações ${ }^{5,9,29}$ não revelaram, ao comparar pacientes com diferentes tipos faciais, diferenças significativas no fluxo aéreo.

A interpretação dos resultados apresentados na tabela 5 comprova o alto grau de significância estatística entre os padrões respiratórios e a variável Profundidade facial, não apresentando diferenças significativas com as demais variáveis. Encontrou-se concordância na literatura ${ }^{5,9}$ de que os padrões respiratórios não exerceram influência sobre as variáveis Altura facial anteroinferior e Ângulo do plano mandibular. Com relação às demais variáveis - Eixo facial e Arco mandibular -, não foi verificada nenhuma correlação citada na revisão de literatura. Inúmeros trabalhos $^{8,11,12,18,27,28}$, contrariamente aos anteriores e aos nossos resultados, caracterizam o aumento da Altura facial anteroinferior, bem como da inclinação do plano mandibular, como alterações que resultam de uma disfunção nasorrespiratória; da mesma forma que foram encontradas concordâncias $^{26}$ nas correlações entre a face longa, a profundidade facial e a respiração bucal.

Baseado nessas afirmações, compreende-se a importância do diagnóstico e tratamento precoces nos pacientes em crescimento, especialmente nos indivíduos com padrão esquelético severo associado ao padrão respiratório predominantemente bucal, justificando os objetivos do presente estudo. 


\section{CONCLUSÕES}

Baseado na metodologia empregada e nos resultados obtidos, julga-se lícito concluir que:

- Não foram observadas diferenças estatisticamente significativas entre os padrões respiratórios (bucal e nasal) e as grandezas relacionadas.

- Das associações entre os tipos faciais (dolicofaciais, mesofaciais e braquifaciais) e as variáveis cefalométricas, constatou-se que: as variáveis cefalométricas Convexidade do ponto A, Altura posterior da face, Profundidade da maxila, Cone facial, Comprimento do corpo e Nasofaringe relacionaram-se significativamente entre os tipos braquifacial e dolicofacial, sendo que a variável Cone facial também se relacionou significativamente entre os tipos braquifacial e mesofacial e entre os tipos mesofacial e dolicofacial.
- Das associações entre os tipos faciais (dolicofaciais, mesofaciais e braquifaciais) e os padrões respiratórios (bucal e nasal), houve correlação estatística entre o tipo braquifacial e o padrão de respiração nasal.

- Das associações entre os padrões respiratórios (bucal e nasal) e as variáveis cefalométricas do Índice Vert, houve significância estatística para a variável Profundidade facial.
Enviado em: outubro de 2006 Revisado e aceito: março de 2009

\title{
Cephalometric study of the changes of the medium and lower thirds of the face in patients with different breathing and facial patterns
}

\begin{abstract}
Aim: To assess the possible relation of respiratory patterns and facial types with linear and angular variables of the Ricketts analysis - CF-N.CF-A, Po-Or.ENA-ENP, CF-Go, CF-Xi.Po-Or, Xi-Pm, N-P.Go-M, A-NP, Po-Or.Na - and the Nasopharynx and Oropharynx linear variables proposed by McNamara; and of the facial types with the respiratory patterns, and the respiratory patterns with the Vert variables. Methods: The sample consisted of 88 lateral teleradiographs of Brazilian leucoderm boys and girls, ranging from 7 to 12 years of age (mean age 10.5 years) of whom 45 were mouth-breathers and 43 nasal breathers. The computerized cephalometric method covered analysis of the variables to determine the facial type, according to Ricketts (Vert Index). After the measurements were taken, assessment of the method error by the Dahlberg formula was applied (mean $=0.52$ ), followed by $t$ Student and chi-square tests $(p<0.05)$ statistical assessments. Results and Conclusions: There were no statistically significant alterations in the variables when related to the respiratory patterns; there were statistically significant differences in the following variables: Convexity of point A, Posterior face height, Maxilla depth, Facial cone, Corpus length and Nasopharynx when related to the brachyfacial and dolicofacial types. The variables Facial cone and Corpus length also presented significant differences between the brachyfacial and mesofacial types, and the variable Facial cone presented significant differences among all the facial types; the brachyfacial type presented significant correlation $(p=0.011)$ with the nasal respiratory pattern; and Facial depth presented statistically significant difference $(p=$ 0.028) when related to the respiratory patterns.
\end{abstract}

Keywords: Cephalometry. Craniofacial growth. Mouth-breathing. Ricketts. McNamara. 


\section{REFERÊNCIAS}

1. ALtMANN, E. B. C. Avaliação do tratamento fonoaudiológico nas cirurgias ortognáticas. In: Fissuras lábio palatinas. 1. ed. São Paulo: Pró-Fono, 1992. p. 409-434.

2. BROADBENT, B. H. A new X-ray technique and its application to Orthodontics. Angle Orthod., Appleton, v. 1, p. 45-66, 1931.

3. DAHLBERG, A. G. Statistical methods for medical and biological studies. London: Bradford and Dickens, 1940.

4. DIFRANCESCO, R. C.; BREGOLA, E. G. P.; PEREIRA, L. S.; LIMA, R. S. A obstrução nasal e o diagnóstico ortodôntico. Rev. Dental Press Ortodon. Ortop. Facial, Maringá, v. 11, n. 1, p. 107-113, jan./fev. 2006.

5. HARTGERINK, D. V.; VIG, P. S. Lower anterior face height and lip incompetence do not predict nasal airway obstruction. Angle Orthod., Appleton, v. 59, no. 1, p. 17-23, Sept. 1989.

6. HARVOLD, E. P.; TOMER, S. B.; VARGERVIK, K.; CHIERICI, G. Primate experiments on oral respiration. Am. J. Orthod. St. Louis, v. 79, no. 4, p. 359-372. Apr. 1981

7. HARVOLD, E. P.; VARGERVIK, K.; CHIERICI, G. Primate experiments on oral sensation and dental malocclusion. Am. J. Orthod., St. Louis, v. 63, p. 494-508, Apr. 1973.

8. KERR, W. J. S.; McWILLIAN, J. S.; LINDER-ARONSON, S. Mandibular form and position related to changed mode of breathing - a-five-year longitudinal study. Angle Orthod., Appleton, v. 59, no. 2, p. 91-96, Sept. 1989.

9. KLUEMPER, G. T.; VIG, O. S.; VIG, K. W. L. Nasorespiratory characteristics and craniofacial morphology. Eur. J. Orthod. Oxford, v. 17, no. 6, p. 491-495, Dec. 1995.

10. LAMPASSO, J. D.; PRESTON, C. B.; TOBIAS, P. V. Cephalometric evaluation and measurement of the upper airway. Semin. Orthod., Philadelphia, v. 10, no. 1, p. 3-15, Mar. 2004

11. LINDER-ARONSON, S. Respiratory function in relation to facial morphology and the dentition. Br. J. Orthod., Oxford, v. 6, no. 2, p. 59-71, Apr. 1979

12. LINDER-ARONSON, S.; WOODSIDE, D. G.; LUNDSTROM, A. Mandibular growth direction following adenoidectomy. Am. J. Orthod., St. Louis, v. 89, no. 4, p. 273-284, Apr. 1986.

13. McNAMARA JR., J. A. A method of cephalometric evaluation. Am. J. Orthod., St. Louis, v. 86, no. 6, p. 449-469, Dec. 1984

14. MICHELOTTI, A.; MARTINA, R.; LAINO, A. Class I malocclusion with severe open bite skeletal pattern treatment. Am. J. Orthod. Dentofacial Orthop., St. Louis, v. 97, no. 5, p. 363-373, May 1990

15. MOSS, M. L.; SALENTIJN, L. The primary role of functional matrices in facial growth. Am. J. Orthod., St. Louis, v. 55 no. 6, p. 556-582, June 1969.

16. MOYERS, R. Ortodontia. 3. ed. Rio de Janeiro: Guanabara Koogan, 1987.

17. NETER, J.; KUTNER, M. H.; NACHTSHEIM, C. J.; WASSERMAN, W. Applied linear statistical models. 4th ed. Chicago: Irvin, 1996.
18. O'RYAN, F S : GALLAGHER D M : LABANC, J. P. EPKER, B $\mathrm{N}$. The relation between nasorespiratory function and dentofacial morphology: a review. Am. J. Orthod., St. Louis, v. 82 no. 5, p. 403-410, Nov. 1982.

19. PADOVAN, B. A. E. Deglutição atípica. Separata do artigo: Reeducação mioterápica nas pressões atípicas da língua. Ortodontia, São Paulo, v. 9, n. 1/2, p. 5-59, 1976.

20. RICKETTS, R. M. A foundation for cephalometric communication. Am. J. Orthod., St. Louis, v. 46, no. 5, p. 330-357, May 1960.

21. RICKETTS, R. M. Respiratory obstruction syndrome. Am. J. Orthod., St. Louis, v. 54, no. 7, p. 495-514, Jully 1968.

22. SAADIA, A. M. Airway obstruction and facial form: a review. J. Pedod., Boston, v. 5, no. 3, p. 222-239, Spring 1981

23. SANTOS-PINTO, C. C. M.; SANTOS-PINTO, P. R.; RAMALLI, E. L.; SANTOS-PINTO, A.; RAVELI, D. B. Espaço nasofaringeano: avaliação pela telerradiografia. Rev. Clin. Ortodon. Dental Press, Maringá, v. 4, n. 6, p. 56-62, 2006

24. SHIMIZU, R. H.; MARUO, H.; BIZETTO, M. S. P.; GUARIZA FILHO, O. Estudo cefalométrico comparativo entre crianças respiradoras bucais e nasais nos diferentes tipos faciais. Rev. Dental Press Ortodon. Ortop. Facial, Maringá, v. 9, n. 1, p. 79-87, jan./fev. 2004.

25. SPIEGEL, M. R. Estatística. São Paulo: McGraw Hill, 1974.

26. TIMMS, D. J.; TRENOUTH, M. J. A quantified comparison of craniofacial form with nasal respiratory function. Am. J. Orthod. Dentofacial Orthop., St. Louis, v. 94, no. 3, p. 216-221, Sept. 1988.

27. TROTMAN, C. A.; McNAMARA JR . J. A.; DIBBETS, J. M.; VAN DER WEELE, L. T. Association of lip posture and the dimensions of the tonsils and sagittal airway with facial morphology. Angle Orthod., Appleton, v. 67, no. 6, p. 425-432 Nov./Dec. 1997

28. VARGERVIK, K.; MILLER, A. J.; CHIERICl, G.; HARVOLD, E.; TOMER, B. S. Morphologic response to changes in neuromuscular patterns experimentally induced by altered modes of respiration. Am. J. Orthod., St. Louis, v. 85, no. 2, p. 115-124, Feb. 1984.

29. VIG, P. S.; VIG, K. W. L.; BECK, F. M.; FIELDS, H. W.; SHANKER $\mathrm{S}$. A longitudinal assessment of upper respiratory function and dentofacial morphology in 8-to-12 year-old children. Semin. Orthod., Philadelphia, v. 10, no. 1, p. 45-53, Mar. 2004.

30. YAMADA, T.; TANNE, K.; MIYAMOTO, K.; YAMAUCHI, K. Influences of nasal respiratory obstruction on craniofacial growth in Young Macaca Fuscata monkeys. Am. J. Orthod. Dentofacial Orthop., St. Louis, v. 111, no. 1, p. 38-43, Jan. 1997

31. WOODSIDE, D. G.; LINDER-ARONSON, S.; LUNDSTRÖM, A.; McWILLIAM, J. Crescimento maxilar e mandibular após alteração do modo respiratório. Ortodontia, São Paulo, v. 27, n. 3, p. 95-114, set./dez. 1994.
Endereço para correspondência

Stella Angélica de Souza Gouveia

Praça Floriano, 55 sala 802

CEP: 20.031-050 - Rio de Janeiro / RJ

E-mail: sasg252@uol.com.br 